\title{
Comparison of Therapeutic Effects and Olfactory Function of Oral Glucocorticoid and Intranasal Glucocorticoid on Chronic Rhinosinusitis Patients with Nasal Polyps
}

\author{
Quan Yang and Min Li \\ Department of Otolaryngology, Suzhou Hospital of Integrated Traditional Chinese and Western Medicine, China
}

\begin{abstract}
Objective: To compare the therapeutic effects and olfactory function of oral glucocorticoid and intranasal glucocorticoid in patients with chronic rhinosinusitis (CRS) with nasal polyps.

Study Design: Experimental study.

Place and Duration of Study: Suzhou Hospital of Integrated Traditional Chinese and Western Medicine, from January 2018 to November 2019.

Methodology: One hundred and twenty-two CRS patients with nasal polyps were randomly divided into Group A and Group B with 61 cases in each group. Group A received local application of glucocorticoid (dexamethasone) after nasal endoscopic surgery. Group B received oral glucocorticoid (prednisone) one week before operation and three weeks after operation. Clinical effects of the two groups were compared.

Results: Total effective rate of Group B was higher than that of Group A $(p=0.023)$, recurrence rate in Group B was lower than that in Group A $(p=0.015)$. Nasal airway resistance and olfactory function scores of Group B were lower than those of Group A at one month, three months and six months after operation (all $p<0.001$ ). There was no difference in the incidence of adverse reactions between the two groups $(p=0.649)$.

Conclusion: Compared with intranasal glucocorticoid, oral glucocorticoid can obviously improve the therapeutic effect and olfactory function of patients, and the postoperative recurrence rate is lower.
\end{abstract}

Key Words: Sinusitis, Nasal polyps, Endoscopic sinus surgery, Glucocorticoid.

How to cite this article: Yang Q, Li M. Comparison of Therapeutic Effects and Olfactory Function of Oral Glucocorticoid and Intranasal Glucocorticoid on Chronic Rhinosinusitis Patients with Nasal Polyps. J Coll Physicians Surg Pak 2021; 31(06):699-702.

\section{INTRODUCTION}

If the symptoms of rhinosinusitis do not completely relieve for more than 12 weeks, it can be called chronic rhinosinusitis (CRS). ${ }^{1,2}$ CRS can be divided into without nasal polyps and with nasal polyps. The treatment of CRS with nasal polyps is more complicated, and the therapeutic effect is worse than that of CRS without nasal polyps, and the recurrence rate is higher. Most CRS patients have olfactory dysfunction. ${ }^{3}$ Olfactory disorders can affect people's visceral reactions, emotional activities, socialfunctions and overall quality of life.

Glucocorticoid is the most basic medicine in the medicine treatment of chronic rhinosinusitis in adults. At present, the research on whether glucocorticoid can improve olfactory dysfunction in CRS patients is full of controversy.

Correspondence to: Min Li, Department of Otolaryngology, Suzhou Hospital of Integrated Traditional Chinese and Western Medicine, China

E-mail:vlvr281@163.com

Received: July 06, 2020; Revised: October 15, 2020;

Accepted: December 05, 2020

DOI: https://doi.org/10.29271/jcpsp.2021.06.699
Some studies have reported that short-term oral glucocorticoid can significantly improve olfactory dysfunction in CRS patients with nasal polyps. ${ }^{4,5}$ Late intranasal application of glucocorticoid can also improve patients' sense of smell. ${ }^{6,7}$ However, one meta-analysis study has pointed out that nasal glucoeorticoid cannot improve subjective or objective olfaction dysfunction in CRS patients with nasal polyps. ${ }^{8}$

The purpose of this study was to compare the therapeutic effects and olfactory function of oral glucocorticoid and intranasal glucocorticoid in patients with chronic rhinosinusitis with nasal polyps.

\section{METHODOLOGY}

This experimental study was conducted at Suzhou Hospital of Integrated Traditional Chinese and Western Medicine, China, from January 2018 to November 2019. This study was approved by the Hospital Ethics Committee. One hundred and twenty-two CRS patients with nasal polyps were selected as the research subjects. The inclusion criteria were that nasal endoscopy before operation, and nasal polyps and viscous or viscous purulent secretions from the middle nasal meatus and olfactory fissure area were found. CTscan of paranasal sinuses within one 
month before operation had shown soft tissue density shadow in nasal cavity, inflammatory lesions in single or multiple paranasal sinuses, and bone thickening of some paranasal sinuses; aged 18-70 years; olfactory dysfunction; no previous history of endoscopic sinus surgery; standardised medicine therapy but the therapeutic effect was not good. The exclusion criteria were patients with a history of benign and malignant nasal tumors; contraindications of hormone application such as diabetes, digestive tract ulcer and glaucoma; surgical contraindications; mental diseases; long-term use of immunosuppressive agents; systemic glucocorticoid application for more than one week within three months before admission; those with fungal sinusitis; pregnant or planned pregnant women and nursing women.

All subjects were randomly divided into Group A and Group B with 61 cases in each group. Group A received local application of glucocorticoid (dexamethasone) after nasal endoscopic surgery. Intravenous drip of antibiotics one day before operation. Endoscopic sinus surgery was performed as Messerklinger's operation, and the head was tilted to one side in supine position. The nasal mucosa was wiped with $1 \%$ lidocaine infiltrated cotton tablet, and the middle nasal meatus was anesthetised. Moreover, $2 \mathrm{ml}$ of $2 \%$ lidocaine was injected into the anterior end of the middle turbinate, the anterior edge of the uncinate process and the lateral side of the attachment of the posterior end of the middle turbinate for full anesthesia. First, the nasal cavity polyp tissue was removed, and then the uncinate process was removed to fully open the ethmoid bubble, and expand the natural opening of the maxillary sinus. The opening of ethmoid sinus, frontal sinus and sphenoid sinus in each group was opened under CT guidance. Vaseline gauze was removed under anterior nasal endoscope on the 2 nd day after operation. The nasal mucosa was contracted with $1 \%$ ephedrine cotton tablets. Within 7 days after operation, the nasal mucosa was contracted with $1 \%$ ephedrine cotton tablets daily, and furazone nasal drops and dexamethasone injection were mixed and dripped nasally, 3 drops on each side, 3 times a day. On the 4th day after operation, the nasal cavity was flushed with normal saline once a day for 3 consecutive days to remove blood scab, pseudomembrane, fiber adhesion and blood clot. After 7 days, the patient was discharged from hospital and advised to wash with normal saline once a week for 5 times; and the dosage of furodexamethasone combined with nasal drops was gradually reduced.

Group B received oral glucocorticoid (prednisone) one week before operation and three weeks after operation. The dose of prednisone was $0.5 \mathrm{mg} / \mathrm{kg} /$ daywhen prednisone was stopped, it was gradually reduced by $5 \mathrm{mg}$ every three days until it was completely stopped. Except that glucocorticoid was not used locally in the nose, the other methods were the same as those in Group A. In order to ensure the stability of the operation quality, all operations were performed by the same senior physician.

Follow-up for six months after operation was conducted to compare the clinical efficacy and recurrence rate between the two groups. The evaluation criteria of clinical efficacy were defined as (i) cure: after treatment, the symptoms such as mucosal edema and purulent secretion of the patient disappeared through nasal endoscopy, and the sinus ostium was observed to be well opened; (ii) improvement: after treatment, symptoms such as mucosal edema and purulent secretions of patients examined by nasal endoscopy were obviously relieved; (iii) ineffective: after treatment, the symptoms of mucosal edema and purulent secretion of the patient were not improved by nasal endoscopy, and symptoms such as sinus ostium atresia and polyp hyperplasia could be observed in nasal cavity. Total effective rate was calculated as (number of cured cases + number of effective cases)/total number of cases $\times$ $100 \%$.

The nasal airway resistance and olfactory function of the two groups were compared before operation, and 1-month, 3month and 6-month after operation. Nasal airway resistance was measured by nasal resistance meter. Olfactory function was detected by Japanese T\&T standard olfactory test method. ${ }^{9}$ The higher the score, the worse the olfactory function, as -1 to 1 indicates normal olfactory function, 1.1 to 2.5 indicates slight decline of olfactory function, 2.6 to 4.0 indicates moderate decline of olfactory function, 4.1 to 5.5 indicates serious decline of olfactory function, and $>5.5$ indicates loss of olfactory function. The occurrence of complications included mild throat irritation and cough, delayed morbid reaction, burning sensation of nose, headache, fever,etc. during treatment was compared between the two groups.

SPSS version 25.0 statistical software was used for analysis. Enumeration data was expressed in $n(\%)$, and Chi-square test was used. Kolmogorov-Smirnov test was used for the evaluation of the normality of quantitative data. Measurement data with normal distribution were expressed by mean $\pm \mathrm{SD} /$ median (IQR) and independent sample t-test / Mann-Whitney U-test was conducted. The $p<0.05$ was statistically significant.

\section{RESULTS}

Among 122 patients with CRS with nasal polyps, 67 (54.92\%) were males and 55 (45.08\%) were females, aged 23-65 $(39.67 \pm 2.14)$ years.

Follow-up for 6 months showed that the total effective rate of Group B was $95.08 \%$ (58 cases), which was higher than that of Group A[81.97\%, (50 cases), $p=0.023$, Table I]. The recurrence rate in Group B was 3.28\% (2 cases), which was lower than that in Group A[16.39\%, (10 cases), $p=0.015$, Tablel].

There was no difference in preoperative nasal airway resistance, and preoperative olfactory function score between the two groups ( $p=0.826,0.629$, Table II). The nasal airway resistance and olfactory function scores of Group B were lower than those of Group A at one month, three months and six months afteroperation (all $p<0.001$, Table II).

The incidence of adverse reactions in Group A was $21.31 \%$ ( 13 cases) and Group B was $18.03 \%$ ( 11 cases). There was no difference in the incidence of adverse reactions between the two groups ( $p=0.649$, Table III). 


Table I: Comparison of therapeutic effect and recurrence rate between the two groups after six months of follow-up.
\begin{tabular}{|l|c|c|}
\hline Index & Group A (n=61) & Group B (n=61) \\
\hline Cure [n(\%)] & $30(49.18)$ & $33(54.10)$ \\
\hline Improvement [n(\%)] & $20(32.79)$ & $25(40.98)$ \\
\hline Ineffective [n(\%)] & $11(18.03)$ & $3(4.92)$ \\
\hline Total effective [n(\%)] & $50(81.97)$ & $58(95.08)$ \\
\hline Recurrence [n(\%)] & $10(16.39)$ & $2(3.28)$ \\
\hline
\end{tabular}

\begin{tabular}{|c|c|c|c|}
\hline Index & Group A ( $n=61)$ & Group B (n=61) & p-value \\
\hline Preoperativenasal airway resistance $(\mathrm{kPa} /(\mathrm{s} \cdot \mathrm{L})$ & $2.96(2.77-3.09)$ & $2.93(2.61-3.20)$ & 0.876 \\
\hline Nasal airway resistanceat 1 month $(\mathrm{kPa} /(\mathrm{s} \cdot \mathrm{L})$ & $1.97(1.84-2.05)$ & $1.46(1.30-1.59)$ & $<0.001$ \\
\hline Nasal airway resistanceat 3 months $(\mathrm{kPa} /(\mathrm{S} \cdot \mathrm{L})$ & $1.39(1.30-1.45)$ & $0.97(0.86-1.06)$ & $<0.001$ \\
\hline Nasal airway resistanceat 6 months $(\mathrm{kPa} /(\mathrm{s} \cdot \mathrm{L})$ & $0.82(0.76-0.85)$ & $0.27(0.24-0.29)$ & $<0.001$ \\
\hline Preoperative olfactory function score & $4.55 \pm 0.29$ & $4.58 \pm 0.43$ & 0.629 \\
\hline Olfactory function scoreat 1 month & $3.31 \pm 0.21$ & $2.49 \pm 0.16$ & $<0.001$ \\
\hline Olfactory function scoreat 3 months & $2.42 \pm 0.16$ & $1.82 \pm 0.12$ & $<0.001$ \\
\hline Olfactory function scoreat 6 months & $1.75 \pm 0.11$ & $1.33 \pm 0.09$ & $<0.001$ \\
\hline
\end{tabular}

\begin{tabular}{|c|c|c|}
\hline Index & Group A $(n=61)$ & Group B $(n=61)$ \\
\hline Mild throat irritation and cough [n(\%)] & $2(3.28)$ & $4(6.56)$ \\
\hline Delayed morbid reaction [n(\%)] & $2(3.28)$ & $3(4.92)$ \\
\hline Burning sensation of nose [n(\%)] & $3(4.92)$ & $2(3.28)$ \\
\hline Headache $[\mathrm{n}(\%)]$ & $4(6.56)$ & $1(1.64)$ \\
\hline Fever $[\mathrm{n}(\%)]$ & $2(3.28)$ & $1(1.64)$ \\
\hline Total complications & $13(21.31)$ & $11(18.03)$ \\
\hline$p$-value & \multicolumn{2}{|c|}{0.649} \\
\hline
\end{tabular}

\section{DISCUSSION}

The human olfaction mainly conducts olfactory signals through the olfactory mucosa inside the upper turbinate and nasal septum. The olfactory mucosa is divided into epithelial layer and lamina propria. Inflammatory cells often infiltrate between the two, and eosinophils are the main cells. However, patients with sinusitis and nasal polyps suffer from nasal mechanical obstruction due to high edema of nasal mucosa, which causes poor nasal ventilation and inability of external olfactory gas to reach the epithelial region of olfactory mucosa, resulting in olfactory dysfunction in patients. ${ }^{10}$

There is no uniform olfactory detection standard at present because of the complexity of olfactory generation and the great influence of psychological factors. At present, olfactory function detection can be divided into subjective detection and objective detection methods. Subjective examination of olfactory function includes subjective symptom scoring method and visual analog scale scoring method. ${ }^{11}$ Methods for objective examination of olfactory function include Japanese T\&T standard olfactory test method, etc. ${ }^{12}$ Some studies have shown that glucocorticoid may improve olfactory function by inhibiting inflammatory reaction, reducing edema and promoting olfactory epithelial regeneration. ${ }^{13}$

The results of this study showed that the olfactory function scores of patients in Group B were lower than those in Group A at one month, three months and six months after operation.
This showed that oral glucocorticoid therapy could significantly improve nasal obstruction; and purulent secretion increase, caused by nasal mucosal edema in patients, improve mucilia function, and be conducive to the recovery of olfactory function in patients. However, some studies have confirmed that intranasal application of glucocorticoid cannot improve olfactory function of patients with chronic rhinosinusitis with nasal polyps. ${ }^{14}$ Some studies have pointed out that short-term oral glucocorticoid can improve olfactory dysfunction in patients with chronic rhinosinusitis with nasal polyps. ${ }^{15}$ The above research conclusions are basically consistent with the research results of this study.

This study also found that the nasal airway resistance of patients in Group B was lower than that in Group A at one month, three months and 6 months after operation. This showed that endoscopic sinus surgery could remove the focus tissue in nasal cavity and paranasal sinuses. This surgery combined with oral glucocorticoid therapy can obviously reduce airway resistance and effectively protect the physiological structure and function of nasal cavity of patients.

As endoscopic sinus surgery can only correct the pathological structures such as fibrosis and polyps in the nasal cavity, and cannot completely remove inflammation in the nasal cavity, postoperative patients may suffer from nasal mucosal edema, adhesion and other symptoms, which is easy to lead to postoperative recurrence. This study confirmed that oral glucocorticoid had lower recurrence rate than intranasal glucocorticoid 
in the treatment of chronic rhinosinusitis patients with nasal polyps. It may be that endoscopic sinus surgery combined with oral glucocorticoid therapy can better play a role in resisting nasal mucosal edema, adhesion and the like, thus reducing the postoperative recurrence rate.

This study found that there was no significant difference in adverse reactions between oral glucocorticoid and intranasal glucocorticoid in the treatment of chronic rhinosinusitis patients with nasal polyps. It should be noted that the monitoring of patients' safety indicators in this study is still insufficient, limited to conditions, bone mineral density has not been measured, and no specialised endocrinologist has been invited to participate in safety evaluation. The long-term and lasting improvement of olfactory function in patients with chronic rhinosinusitis, accompanied by nasal polyps treated by oral glucocorticoid, needs further research to confirm.

\section{CONCLUSION}

Compared with intranasal glucocorticoid, oral glucocorticoid can obviously improve the therapeutic effect and olfactory function of patients, and the postoperative recurrence rate becomes lower.

\section{ETHICAL APPROVAL:}

This study was conducted with the approval from the Ethics Committee of Suzhou Hospital of Integrated Traditional Chinese and Western Medicine, China.

\section{PATIENTS' CONSENT:}

All patients signed a document of informed consent.

\section{CONFLICT OF INTEREST:}

The authors declared no conflict of interest.

\section{AUTHORS' CONTRIBUTION:}

QY: Acquired data, drafted the manuscript and contributed substantially to its revision.

ML: Acquired data, contributed substantially to its revision and approved the final manuscript.

\section{REFERENCES}

1. Kennedy DW. Chronic rhinosinusitis (CRS). Int Forum Allergy Rhinol 2015; 5(8):665-6. doi: 10.1002/alr.21624.

2. Cho SH, Hamilos DL, Han DH, Laidlaw TM. Phenotypes of chronic rhinosinusitis. J Allergy Clin Immunol Pract 2020; 8(5):1505-11. doi: 10.1016/j.jaip.2019.12.021.

3. Mattos JLF. Mechanisms and treatment of olfactory dysfunction in chronic rhinosinusitis. Ann Allergy Asthma Immunol 2020; 124(4):307-8. doi: 10.1016/j.anai.2020. 01.014.

4. Alobid I, Benitez P, Valero A, Munoz R, Langdon C, Mullol J. Oral and intranasal steroid treatments improve nasal patency and paradoxically increase nasal nitric oxide in patients with severe nasal polyposis. Rhinology 2012; 50(2):171-7. doi: 10.4193/Rhin10.140.

5. Alobid I, Benítez P, Cardelús S, de Borja Callejas F, Lehrer-Coriat $E$, Pujols $L$, et al. Oral plus nasal corticosteroids improve smell, nasal congestion, and inflammation in sino-nasal polyposis. Laryngoscope 2014; 124(1):50-6. doi: 10.1002/lary. 24330.

6. Small CB, Hernandez J, Reyes A, Schenkel E, Damiano A, Stryszak $P$, et al. Efficacy and safety of mometasone furoate nasal spray in nasal polyposis. J Allergy Clin Immunol 2005; 116(6):1275-81. doi: 10.1016/j.jaci. 2005.07.027.

7. Jankowski R, Klossek JM, Attali V, Coste A, Serrano E. Longterm study of fluticasone propionate aqueous nasal spray in acute and maintenance therapy of nasal polyposis. Allergy 2009; 64(6):944-50. doi: 10.1111/j.1398-9995. 2009.01938.x.

8. Li ZW, Guo JY, Zhou J, Yan FB, Yang ZM, Ding ZH. Meta-analysis of the olfaction effectiveness of glucocorticoid in the management of chronic rhinosinusitis with nasal polyposis. J Clin otorhinolaryngol Head Neck Surg (China) 2015; 29(1):1868-72.

9. Georgalas C, Cornet M, Adriaensen G, Reinartz S, Holland C, Prokopakis $\mathrm{E}$, et al. Evidence-basedsurgery for chronic rhinosinusitis with and without nasalpolyps. Current Allergy and Asthma Reports 2014; 14(4):1-7. doi: 10.1007/s11882014-0427-7.

10. Gelardi M, Piccininni K, Quaranta N, Quaranta V, Silvestri M, Ciprandi G. Olfactory dysfunction in patients with chronic rhinosinusitis with nasal polyps is associated with clinical-cytological grading severity. Acta Otorhinolaryngol Ital 2019; 39(5):329-35. doi: 10.14639/0392-100X-2426.

11. Kim BY, Kang SG, Kim SW, Hong YK, Jeun SS, Kim SW, et al. Olfactory changes after endoscopic endonasal transsphenoidal approach for skull base tumors. Laryngoscope 2014; 124(11):2470-5. doi: 10.1002/lary. 24674.

12. Shiga H, Taki J, Okuda K, Watanabe N, Tonami H, Nakagawa $\mathrm{H}$, et al. Prognostic value of olfactory nerve damage measured with thallium-based olfactory imaging in patients with idiopathic olfactory dysfunction. Sci Rep 2017; 7(1):3581. doi: 10.1038/s41598-017-03894-4.

13. Seo BS, Lee HJ, Mo JH, Lee CH, Rhee CS, Kim JW. Treatment of postviral olfactory loss with glucocorticoids, Ginkgo biloba, and mometasone nasal spray. Arch Otolaryngol Head Neck Surg 2009; 135(10):1000-4. doi: 10.1001/ archoto.2009.141.

14. Ehnhage A, Olsson P, Kölbeck KG, Skedinger M. Functional endoscopic sinus surgery improved asthma symptoms as well as PEFR and olfaction in patients with nasal polyposis. Allergy 2009; 64(5):762-9. doi: 10.1111/j.1398-9995.2008. 01870.x.

15. Poetker DM, Jakubowski LA, Lal D, Hwang PH, Wright ED, Smith TL. Oral corticosteroids in the management of adult chronic rhinosinusitis with and without nasal polyps: an evidence-based review with recommendations. Int Forum Allergy Rhinol 2013; 3(2):104-20. doi: 10.1002/alr.21072. 